\title{
Endoscopic Ultrasound-Guided, Percutaneous, and Transjugular Liver Biopsy: A Comparative Systematic Review and Meta-Analysis
}

\author{
Thomas R. McCarty ${ }^{1,2}$, Ahmad Najdat Bazarbashi ${ }^{1,2}$, Basile Njei ${ }^{3}$, Marvin Ryou ${ }^{1,2}$, Harry R. Aslanian ${ }^{3}$ and Thiruvengadam Muniraj ${ }^{3}$ \\ ${ }^{1}$ Division of Gastroenterology, Hepatology and Endoscopy, Brigham and Women's Hospital, Boston, MA, ${ }^{2}$ Harvard Medical School, \\ Boston, MA, ${ }^{3}$ Section of Digestive Diseases, Yale University School of Medicine, New Haven, CT, USA
}

Background/Aims: Percutaneous liver biopsy (PCLB) or transjugular liver biopsy (TJLB) have traditionally been performed to obtain a sample of hepatic tissue; however, endoscopic ultrasound-guided liver biopsy (EUSLB) has become an attractive alternative. The aim of this study was to compare the efficacy and safety of EUSLB, PCLB, and TJLB.

Methods: Search strategies were developed in accordance with PRISMA and MOOSE guidelines. Major outcomes included the following: adequacy of biopsy specimens (i.e., complete portal triads [CPT], total specimen length [TSL] in mm, and length of longest piece [LLP]) in $\mathrm{mm}$ ), and rate of adverse events. Only studies comparing all biopsy approaches (i.e., EUSLB, PCLB, and TJLB) were included.

Results: Five studies (EUSLB [ $n=301]$; PCLB [ $n=176]$; and TJLB [ $n=179]$ ) were included. Biopsy cumulative adequacy rates for EUSLB, PCLB, and TJLB were 93.51\%, 98.27\%, and 97.61\%, respectively. Based on the subgroup analysis limited to EUS biopsy needles in current clinical practice, there was no difference in biopsy adequacy or adverse events for EUSLB compared to PCLB and TJLB (all $p>0.050$ ). A comparison of EUSLB and PCLB revealed no difference between specimens regarding both CPT ( $p=0.079)$ and LLP ( $p=0.085)$; however, a longer TSL $(p<0.001)$ was observed. Compared to TJLB, EUSLB showed no difference in LLP ( $p=0.351)$, fewer CPT ( $p=0.042)$, and longer TSL $(p=0.005)$.

Conclusions: EUSLB appears to be a safe, minimally invasive procedure that is comparable to PCLB and TJLB regarding biopsy specimens obtained and rate of adverse events associated with each method. Clin Endosc 2020;53:583-593

Key Words: Endoscopic ultrasound (EUS); Interventional endoscopy; Liver biopsy

\section{INTRODUCTION}

Histopathologic examination of liver tissue obtained via biopsy may provide essential diagnostic and clinical information impacting patient care. ${ }^{1-4}$ Traditionally, hepatic tissue

Received: November 11, 2019 Revised: January 27, 2020

Accepted: April 3, 2020

Correspondence: Thiruvengadam Muniraj

Section of Digestive Diseases, Yale University School of Medicine, 333 Cedar Street, LMP 1080, New Haven, CT 06520, USA

Tel: +1-203-785-7312, Fax: +1-203-785-7273, E-mail: thiruvengadam.muniraj@ yale.edu

ORCID: https://orcid.org/0000-0002-4904-2645

This work was previously submitted to Digestive Disease Week 2019 with the abstract published as Su1157 EUS-guided, percutaneous, and transjugular liver biopsy: a comparative systematic review and meta-analysis, Gastrointestinal Endoscopy, 2019.

(c) This is an Open Access article distributed under the terms of the Creative Commons Attribution Non-Commercial License (http://creativecommons.org/ licenses/by-nc/3.0) which permits unrestricted non-commercial use, distribution, and reproduction in any medium, provided the original work is properly cited. specimens have been obtained via a percutaneous, transjugular, or surgical approach. Dr. Paul Ehrlich is credited with the first liver aspiration in 1883. Subsequently, both Dr. Bingle in 1923 and Dr. Menghini in 1958 implemented several modifications to the technique before becoming widely accepted. ${ }^{1,3-6}$ The transvenous approach, typically through the transjugular route, followed a few years later in 1964 .

While advancements in medical technology, especially imaging resolution and elastography, have reduced the role of liver biopsy as standard practice for many individuals with liver disease, histologic examination of hepatic tissue is critical for staging of parenchymal liver disease, development of treatment plans based upon histologic analysis, and diagnostic evaluation of many acute and chronic liver abnormalities. ${ }^{8}$ For a majority of patients, percutaneous liver biopsy (PCLB) remains the preferred approach since it is less invasive and low cost compared with the transjugular route. ${ }^{6,8}$ However, transjugular liver biopsy (TJLB) may be preferred for patients with 
coagulopathy, large-volume ascites, or a suspected vascular tumor. TJLB is also commonly considered a potential alternative for patients in whom the percutaneous approach is suboptimal, contraindicated, or has been previously unsuccessful. ${ }^{1,9,10}$ The transjugular approach may also be more feasible in patients with acute liver failure with associated coagulopathy, or among patients with cirrhosis that would benefit from hepatic venous pressure gradient measurement to assess for clinically significant portal hypertension. ${ }^{11}$

Endoscopic ultrasound-guided liver biopsy (EUSLB) has become an attractive alternative to percutaneous and transjugular approaches. Potential advantages of this technique include real-time imaging during the entire procedure, acquisition of liver biopsy specimens simultaneously during endosonography, greater accessibility to multiple sites (i.e., left lobe, caudate lobe, and right lobe) regardless of body habitus, improved accessibility to small liver lesions, and avoidance of invasive procedures, such as vascular puncture or catheter placement. ${ }^{12-14}$ While an EUS-guided transgastric approach offers a novel method for obtaining liver biopsies in patients undergoing upper endosonography, there is a paucity of data to compare the outcomes of various biopsy strategies. The primary aim of this study was to perform a structured systematic review and meta-analysis to compare the efficacy and safety of EUSLB, PCLB, and TJLB.

\section{MATERIALS AND METHODS}

\section{Study design and search strategy}

The guidelines of the Preferred Reporting Items for Systematic Reviews and Meta-Analyses (PRISMA) and the Meta-Analysis of Observational Studies in Epidemiology (MOOSE) for reporting systematic reviews and meta-analyses were followed based on an established protocol-the PRISMA and MOOSE checklists are provided as Supplementary materials 1 and $2 \cdot{ }^{15,16}$ Also, this study was submitted to PROSPERO, an international database of prospectively registered systematic reviews in health and social care. Individualized search strategies were developed for the following databases from inception through November 30, 2018: PubMed, EMBASE, Web of Science, and Cochrane databases. The medical subject heading (MeSH) terms included: liver biopsy. For articles related to liver biopsy, subject heading search terms and title and abstract were reviewed for the following: endoscopic ultrasound (EUS), percutaneous, transvenous, or transjugular.

All relevant articles, regardless of type, year of publication, or status (full-text manuscripts and published abstracts), were included. Based on the initial search results, the titles and abstracts of all potential studies were screened for eligibility, with references manually reviewed by cross-checking bibliographies of retrieved articles. Two reviewers (TRM and ANB) independently performed the screening process of titles and abstracts according to predefined inclusion and exclusion criteria. Any differences between TRM and ANB were resolved by mutual agreement and in consultation with a third reviewer (BN). For studies with incomplete information, primary contact was attempted with the principal authors to obtain missing data.

\section{Study selection criteria}

Randomized controlled trials and observational studies evaluating all three treatment cohorts (EUSLB, PCLB, and TJLB) were included in this analysis. For comparative review, we included studies with adults patients aged 18 and older, and studies that used all three techniques (EUSLB, PCLB, and TJLB) for hepatic sampling. Included studies were required to report at least one of the primary outcomes for study analysis. Non-human studies, or studies that only compared two modalities, were excluded. Multiple published works from similar authors were evaluated for overlapping enrollment times to preserve independence of observations, with contact of principal authors performed when needed. Studies with insufficient data, as well as study types that did not report primary or independent data, were excluded from this systematic review and meta-analysis. Case series were also not included in effort to reduce selection bias.

\section{Endoscopic ultrasound-liver biopsy needle type}

All EUSLB needle types were included for cumulative analysis. However, on subgroup analysis, only needles used in routine clinical practice were considered for inclusion. The available EUSLB needle types that were considered acceptable for subgroup analysis included the $19 \mathrm{G}$ fine needle aspiration (FNA) or the fine needle biopsy (FNB) - including $19 \mathrm{G}$ SharkCore FNB (Medtronic Co., Minneapolis, MN, USA), 19 G Expect FNA (Boston Scientific, Natick, MA, USA), 22 G SharkCore FNB needle, and the 19 G, 22 G, and 25 G Acquire EUS FNB needle (Boston Scientific). Based on previous comparator studies, older or first generation EUS core needles, including needles that are no longer available in clinical practice, were excluded to simulate real-world clinical practice. ${ }^{17-20}$ The excluded needle types comprised the 19 G or 22 G ProCore, 18 G QuickCore (Cook Medical, Bloomington, IN, USA), and Coaxial Temno needle (Care-Fusion, McGraw Park, IL, USA).

\section{Outcome measures}

The primary outcomes of this structured systematic review and meta-analysis were adequacy of biopsy specimens for pathology review (i.e., complete portal triads [CPT], total 
specimen length [TSL] in mm, and length of longest piece [LLP] in $\mathrm{mm}$ ), and rate of adverse events. The adequacy of a liver biopsy has conventionally been defined in terms of the minimum length of the tissue cores and the presence of a minimum number of complete portal tracts (defined as at least 6 to 11 complete portal tracts for adequate diagnosis). ${ }^{1,21,22}$ Previous systematic reviews and meta-analyses of percutaneous and transjugular routes to obtain hepatic tissue have defined adequacy of liver biopsy as 7.5 CPT, with TSL of $17.7 \mathrm{~mm}$, and 6.5 CPT, with TSL of $12 \mathrm{~mm}$, respectively. ${ }^{10,23}$ Currently, an optimal definition of specimen adequacy for EUSLB has not yet been established. Adverse events were primarily concerned with bleeding; however, direct procedure-related complications, such as significant abdominal pain, were also included.

\section{Statistical analysis}

The systematic review and meta-analysis were performed using pooled proportions. Subsequently, individual study proportions were transformed into a quantity using the Freeman-Tukey variant of the arcsine square root transformed proportion. Next, pooled proportions were calculated as the back transform of the weighted mean of the transformed proportion using DerSimonian-Laird weights for the random effects model. ${ }^{24-27}$ The rates were estimated using a random effects model and then presented as point estimates (rates) with $95 \%$ confidence intervals (CIs). ${ }^{28-30}$

Subgroup analyses were also performed based upon the comparative effectiveness of varying biopsy strategies limited to studies with EUS biopsy needles used in current clinical practice. If there were $<3$ studies included, pooled proportions using a random effects model could not be calculated. In this case, non-pooled outcome measures were used to compare outcomes between different sampling strategies using two-sample $t$-tests for binomial proportions. All calculated $p$-values were 2 -sided, and a $p$-value of $<0.05$ was considered statistically significant. Tabular and graphical analyses were performing using Comprehensive Meta-Analysis software, version 3 (BioStat, Englewood, NJ, USA). Combined weighted proportions were determined by use of the Stata 15.0 software package (StataCorp LP, College Station, TX, USA).

\section{Risk of bias and quality assessment}

For observational studies, the risk of bias and quality were evaluated using the Newcastle-Ottawa Quality Assessment Scale. For randomized controlled trials, the JADAD score was used to determine quality. ${ }^{31,32}$ High-quality studies were defined as a Newcastle-Ottawa Quality Assessment Scale score of $\geq 5$ or a JADAD score of $\geq 4$. For observational studies, scores were determined by study design, full manuscript or abstract article type, cohort size, specimen collection details, histologic examination, adverse event reporting, and attrition rate similar to previously published literature. ${ }^{20}$ Risk of bias and quality of studies were assessed independently by two authors (TRM and ANB), with disagreements resolved by consultation with the third reviewer (BN).

\section{Investigations of heterogeneity}

Heterogeneity was assessed using the chi squared test and the $I^{2}$ statistic. ${ }^{33}$ Significant heterogeneity was defined as $p<0.05$ using the Cochran Q test or $I^{2}>50 \%$, with values $>50 \%$ indicating substantial heterogeneity. Based on the use of the random effects model to estimate average effect size, a 95\% prediction interval was calculated to determine the dispersion of effects and clearly illustrate heterogeneity in the calculated effect size. ${ }^{28,34-36}$

\section{Publication bias}

To assess publication bias, Egger regression testing was used and a funnel plot was created and visually inspected for asymmetry. ${ }^{37,38}$ The trim and fill method was used to correct for funnel plot asymmetry and to provide an adjusted effect. ${ }^{39}$ The classic fail-safe test was also applied to assess risk of bias across studies.

\section{RESULTS}

\section{Included study and patient characteristics}

Five comparator studies were included in this meta-analysis and a total of 656 patients were enrolled-EUSLB $(n=301)$; PCLB $(n=176)$; and TJLB $(n=179) .{ }^{14,40-43}$ A PRISMA flow chart of search results is shown in Fig. 1. Three abstracts and two full-text manuscripts published between 2015 and 2018 were included in this analysis. Among the included studies, four were retrospective in design and one was prospective. The cumulative data of all studies and patient characteristics are highlighted in Table 1. The mean age of all included patients was $51.62 \pm 2.81$ years and $43.93 \%$ of patients were male. The adequacy rate of biopsy specimens for EUSLB was $93.51 \%$ (95\% CI, 60.41 to 99.27); $I^{2}=88.40 \%$; prediction interval -1.00 to 1.00 , range 2.00; Meanwhile, for PCLB, the adequacy rate was $98.27 \%$ (95\% CI, 93.31 to 99.57 ); $I^{2}=0.00 \%$; prediction interval 0.68 to 1.00 , range 0.32 . Finally, for TJLB, the adequacy rate was $97.61 \%$ ( $95 \% \mathrm{CI}, 93.39$ to 99.16$) ; I^{2}=0.00 \%$; prediction interval 0.78 to 1.00 , range 0.22 (Fig. $2 \mathrm{~A}-\mathrm{C}$ ). The cumulative data for each modality is highlighted in Supplementary Table 1.

\section{Comparative subgroup analyses}

In order to compare the three modalities, only EUS biopsy 

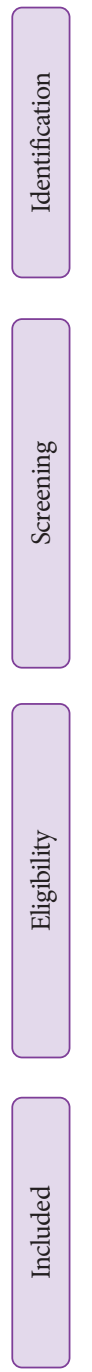

Electronic database search $(n=29,162)$

PubMed $(n=10,995)$

Embase $(n=7,128)$

Web of Science $(n=10,399)$

Cochrane $(n=640)$

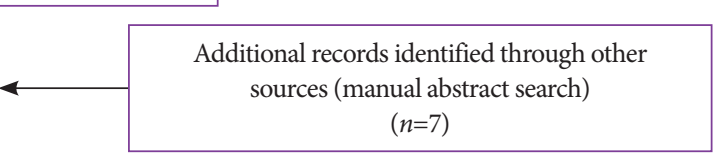

Duplicates excluded $(n=15,345)$

Records after duplicates removed $(n=13,824)$

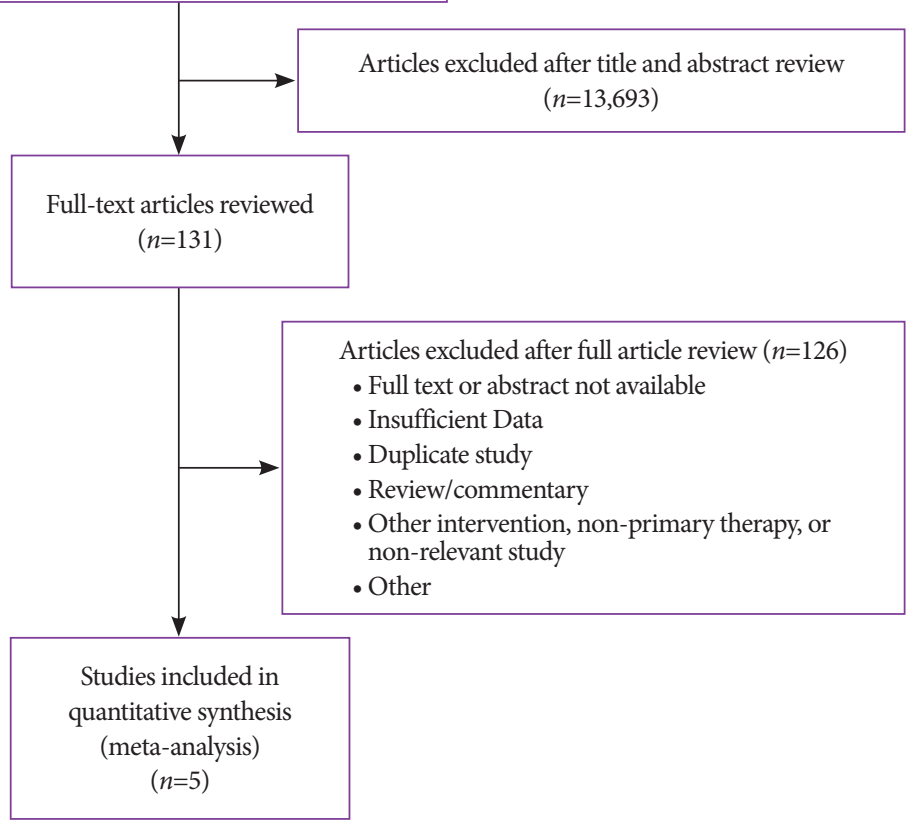

Fig. 1. Preferred reporting items for systematic reviews and meta-analyses (PRISMA) flow chart of search results.

needles utilized in current clinical practice were considered. Indeed, the study by Nakanishi et al. was excluded given inclusion of needle types not routinely utilized in clinical practice. ${ }^{14}$ Regarding adequacy of biopsy specimens, EUSLB was comparable to PCLB ( $98.53 \%$ vs. $100.00 \%, p=0.410)$ and was similar to TJLB ( $98.53 \%$ vs. $100.0 \%, p=0.351$ ). Meanwhile, adverse events with EUSLB were similar to PCLB (16.46\% vs. $12.24 \%, p=0.516$ ) and to TJLB (EUS: $16.46 \%$ vs. TJLB: $4.88 \%$, $p=0.071)$.

Analysis of specimen adequacy also involved comparison of CPT, TSL, and LLP among the three modalities. A comparison of EUSLB and PCLB resulted in histologic samples with no difference in CPT (odds ratio [OR], 4.70; 95\% CI, -0.54 to
9.92; $I^{2}=79.79 \% ; p=0.079 ;$ Fig. $\left.3 \mathrm{~A}\right)$, no difference in LLP (OR, -3.34 ; $95 \%$ CI, -7.14 to $0.46 ; I^{2}=79.63 \%$; $p=0.085$; Fig. $\left.3 \mathrm{~B}\right)$, and longer TSL (OR, 11.35; 95\% CI, 6.77 to $15.93 ; I^{2}=0.00 \%$; $p<0.001$; Fig. 3C). Meanwhile, a comparison of EUSLB and TJLB resulted in no difference in LLP $(-1.92$ [95\% CI, -5.94 to 2.11]; $I^{2}=82.86 \%$; $p=0.351$; Fig. $4 \mathrm{~A}$ ), smaller number of CPT -1.67 ( $95 \%$ CI, -3.28 to $-0.06 ; I^{2}=0.00 \%$; $p=0.042$; Fig. $4 \mathrm{~B}$ ), and longer TSL (OR, 6.28; 95\% CI, 1.93 to $10.63 ; I^{2}=3.94 \%$; $p=0.005$; Fig. 4C).

\section{Risk of bias assessment}

All 5 studies were assessed using the Newcastle-Ottawa Quality Assessment Scale, which assigned a high-quality 


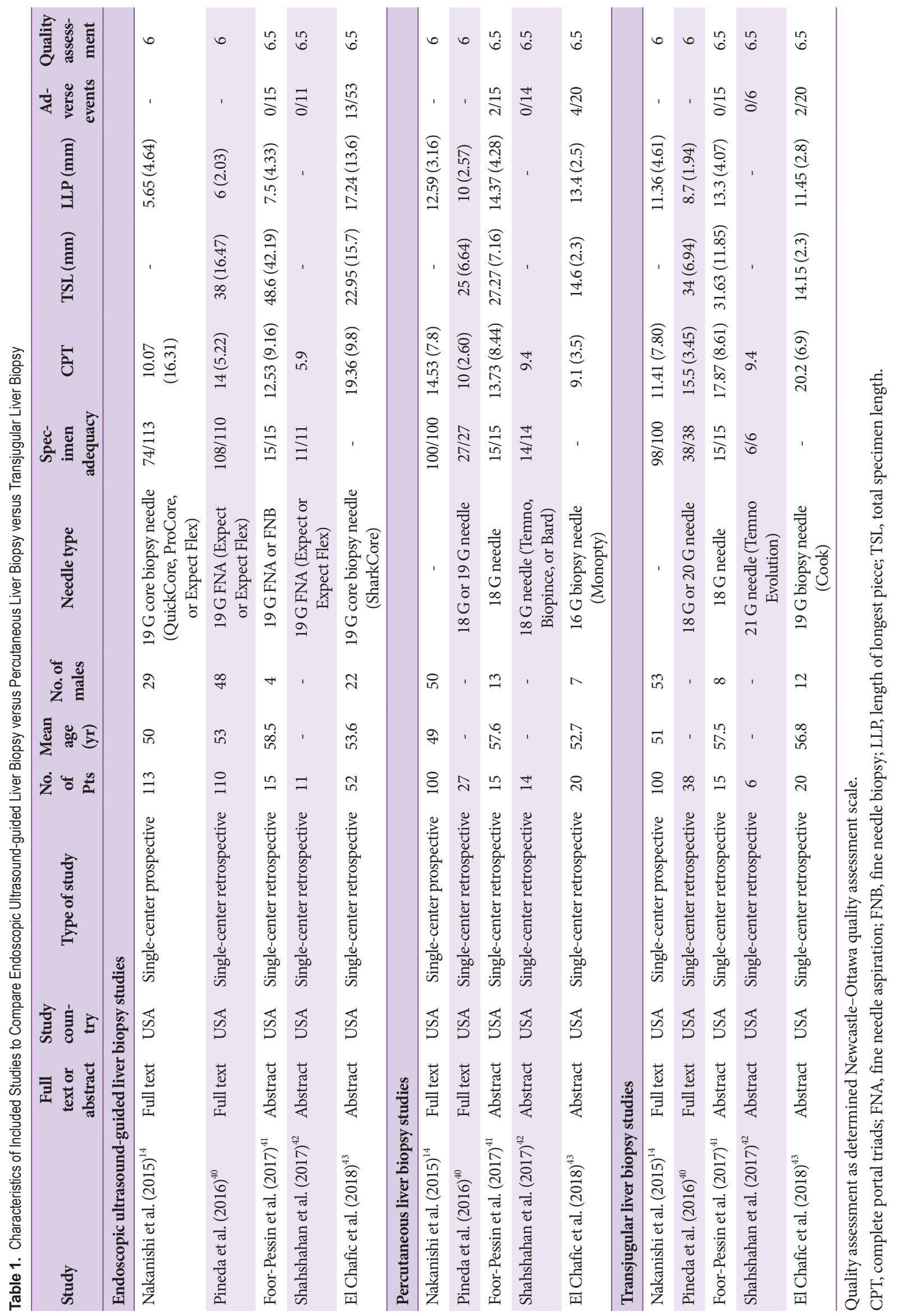


Adequacy of endoscopic ultrasound liver biopsy - cumulative data

\begin{tabular}{lccccc} 
Study & \multicolumn{5}{c}{ Statistics for each study } \\
& $\begin{array}{c}\text { Event } \\
\text { rate }\end{array}$ & $\begin{array}{c}\text { Lower } \\
\text { limit }\end{array}$ & $\begin{array}{c}\text { Upper } \\
\text { limit }\end{array}$ & Z-value & $p$-value \\
Foor-Pessin et al. (2017) & 0.9688 & 0.6497 & 0.9981 & 2.3900 & 0.0169 \\
Shahshahan et al. $(2017)^{42}$ & 0.9583 & 0.5754 & 0.9974 & 2.1704 & 0.0300 \\
Pineda et al. (2016) & 0.9818 & 0.9302 & 0.9954 & 5.5898 & 0.0000 \\
Nakanishi et al. (2015) & 0.6549 & 0.5628 & 0.7366 & 3.2369 & 0.0012 \\
& 0.9351 & 0.6041 & 0.9927 & 2.3288 & 0.0199
\end{tabular}

(A)

(B) $\underline{\text { Study }}$ Shahshahan et al. (2017) Pineda et al. (2016)
Foor-Pessin et al. (2017) $)^{41}$ Nakanishi et al. $(2015)^{14}$$$
\text { rate limit limit Z-value } p \text {-value }
$$$$
\begin{array}{llllll}
0.9688 & 0.6497 & 0.9981 & 2.3900 & 0.0169
\end{array}
$$$$
\begin{array}{lllll}
0.9667 & 0.6337 & 0.9979 & 2.3410 & 0.0192
\end{array}
$$$$
\begin{array}{lllll}
0.9821 & 0.7704 & 0.9989 & 2.8082 & 0.0050
\end{array}
$$$$
\begin{array}{lllll}
0.9950 & 0.9258 & 0.9997 & 3.7407 & 0.0002
\end{array}
$$$$
\begin{array}{lllll}
0.9827 & 0.9331 & 0.9957 & 5.6462 & 0.0000
\end{array}
$$

B)

Adequacy of percutaneous liver biopsy - cumulative data

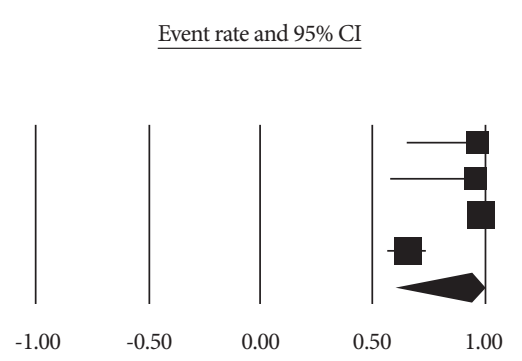

Adequacy of transjugular liver biopsy - cumulative data

\begin{tabular}{|c|c|c|c|c|c|c|c|c|c|c|}
\hline \multirow[t]{2}{*}{$\underline{\text { Study }}$} & \multicolumn{5}{|c|}{ Statistics for each study } & & \multicolumn{3}{|c|}{ Event rate and 95\% CI } & \\
\hline & $\begin{array}{l}\text { Event } \\
\text { rate }\end{array}$ & $\begin{array}{l}\text { Lower } \\
\text { limit }\end{array}$ & $\begin{array}{l}\text { Upper } \\
\text { limit }\end{array}$ & Z-value & $p$-value & & & & & \\
\hline Foor-Pessin et al. $(2017)^{41}$ & 0.9688 & 0.6497 & 0.9981 & 2.3900 & 0.0169 & & & & & \\
\hline Shahshahan et al. $(2017)^{42}$ & 0.9286 & 0.4228 & 0.9957 & 1.7477 & 0.0805 & & & & & - \\
\hline Pineda et al. $(2016)^{40}$ & 0.9872 & 0.8255 & 0.9992 & 3.0518 & 0.0023 & & & & & \\
\hline Nakanishi et al. $(2015)^{14}$ & 0.9800 & 0.9236 & 0.9950 & 5.4485 & 0.0000 & & & & & \\
\hline & 0.9761 & 0.9339 & 0.9916 & 6.8453 & 0.0000 & & & & & \\
\hline & & & & & & -1.00 & -0.50 & 0.00 & 0.50 & 1.00 \\
\hline
\end{tabular}

(C)

Meta analysis

Fig. 2. (A) Cumulative liver biopsy adequacy for endoscopic ultrasound-guided liver biopsy. (B) Cumulative liver biopsy adequacy for percutaneous liver biopsy. (C) Cumulative liver biopsy adequacy for transjugular liver biopsy. $\mathrm{Cl}$, confidence interval.

grade (i.e., score $\geq 5$ ) to the five studies (four retrospective and one prospective). Authors' judgements each included study is highlighted in Table 1. Publication bias was also assessed. Visual inspection of the funnel plot for studies assessing adequacy demonstrated that smaller and statistically insignificant studies appeared to be missing likely due to publication bias (Supplementary Fig. 1A). Adequacy of EUSLB was decreased from $93.51 \%$ (95\% CI, 60.41 to 99.27 ) to $81.94 \%$ (95\% CI, 47.49 to 95.79) with Duval and Tweedie's trim and fill method (Supplementary Fig. 1B). Using the classic fail-safe test to assess for publication bias, it was determined it would take 43 non-significant studies to nullify the results of this analysis.

\section{DISCUSSION}

Although the need for liver biopsy has largely been replaced in a multitude of hepatic diseases due to the availability of less invasive modalities (i.e., elastography, computed tomography, and magnetic resonance imaging), histologic examination of 


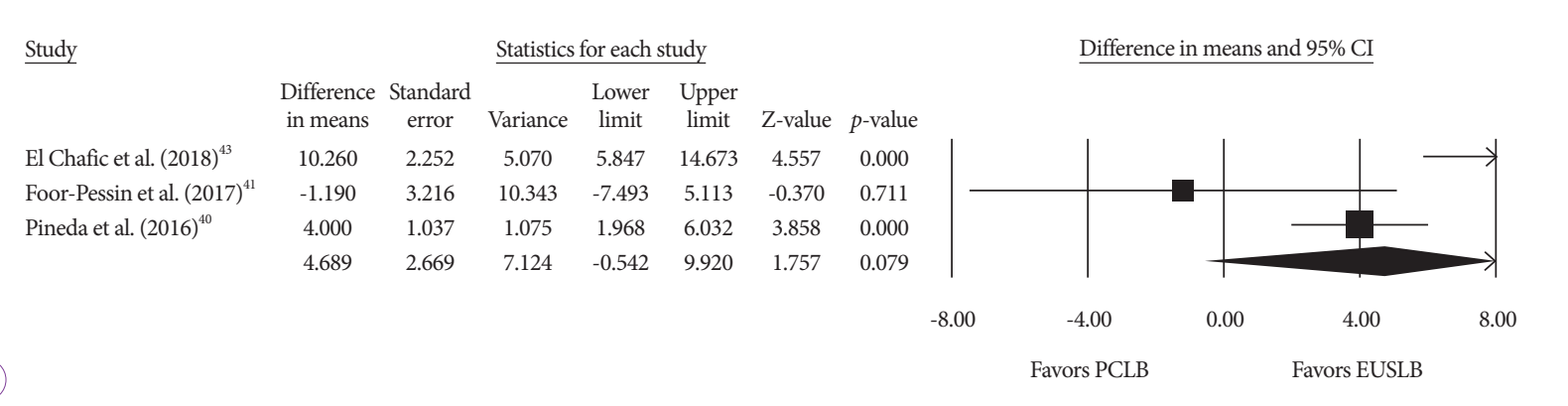

Length of longest piece: EUSLB vs. PCLB

\begin{tabular}{|c|c|c|c|c|c|c|c|c|c|c|c|}
\hline \multirow[t]{2}{*}{ Study } & \multicolumn{7}{|c|}{$\underline{\text { Statistics for each study }}$} & \multicolumn{4}{|c|}{ Difference in means and $95 \% \mathrm{CI}$} \\
\hline & $\begin{array}{l}\text { Difference } \\
\text { in means }\end{array}$ & $\begin{array}{l}\text { Standard } \\
\text { error }\end{array}$ & Variance & $\begin{array}{l}\text { Lower } \\
\text { limit }\end{array}$ & $\begin{array}{l}\text { Upper } \\
\text { limit }\end{array}$ & Z-value & $p$-value & & & & \\
\hline El Chafic et al. $(2018)^{43}$ & 3.840 & 3.073 & 9.444 & -2.183 & 9.863 & 1.250 & 0.211 & & & & \\
\hline Foor-Pessin et al. $(2017)^{41}$ & -6.870 & 1.572 & 2.471 & -9.951 & -3.789 & -4.370 & 0.000 & & & & \\
\hline \multirow[t]{4}{*}{ Pineda et al. $(2016)^{40}$} & -4.000 & 0.461 & 0.212 & -4.903 & -3.097 & -8.684 & 0.000 & & & & \\
\hline & -3.340 & 1.940 & 3.764 & -7.143 & 0.463 & -1.721 & 0.085 & & & & \\
\hline & & & & & & & -8.00 & -4.00 & 0.00 & 4.00 & 8.00 \\
\hline & & & & & & & & Favors $\mathrm{P}$ & & $\mathrm{rs} \mathrm{EU}$ & \\
\hline
\end{tabular}

Total specimen length: EUSLB vs. PCLB

\begin{tabular}{|c|c|c|c|c|c|c|c|c|c|c|c|}
\hline \multirow[t]{2}{*}{$\underline{\text { Study }}$} & \multicolumn{7}{|c|}{$\underline{\text { Statistics for each study }}$} & \multicolumn{4}{|c|}{ Difference in means and $95 \% \mathrm{CI}$} \\
\hline & $\begin{array}{l}\text { Difference } \\
\text { in means }\end{array}$ & $\begin{array}{l}\text { Standard } \\
\text { error }\end{array}$ & Variance & $\begin{array}{l}\text { Lower } \\
\text { limit }\end{array}$ & $\begin{array}{l}\text { Upper } \\
\text { limit }\end{array}$ & Z-value & $p$-value & & & & \\
\hline El Chafic et al. $(2018)^{43}$ & 8.350 & 3.540 & 12.530 & 1.412 & 15.288 & 2.359 & 0.018 & & & & $y$ \\
\hline Foor-Pessin et al. $(2017)^{41}$ & 21.330 & 11.049 & 122.084 & -0.326 & 42.986 & 1.930 & 0.054 & & & & \\
\hline \multirow[t]{4}{*}{ Pineda et al. $(2016)^{40}$} & 13.000 & 3.240 & 10.495 & 6.651 & 19.349 & 4.013 & 0.000 & & & & $\rightarrow$ \\
\hline & 11.348 & 2.336 & 5.456 & 6.769 & 15.926 & 4.858 & 0.000 & & & & $\lambda$ \\
\hline & & & & & & & -8.00 & -4.00 & 0.00 & 4.00 & 8.00 \\
\hline & & & & & & & & Favors PCLB & & Favors EUSLB & \\
\hline
\end{tabular}

Meta analysis

Fig. 3. (A) Forest plot: comparison of complete portal triads for endoscopic ultrasound-guided liver biopsy (EUSLB) versus percutaneous liver biopsy (PCLB). (B) Forest plot: comparison of length of longest piece for EUSLB versus PCLB. (C) Forest plot: comparison of total specimen length for EUSLB versus PCLB. Cl, confidence interval.

hepatic tissue is still required for many diagnoses and may be pivotal to determine appropriate treatments. Based on the results of this systematic review and meta-analysis, adequacy of hepatic specimen and rate of adverse events were comparable between EUSLB, PCLB, and TJLB.

\section{Advantages and disadvantages of endoscopic ultrasound-guided liver biopsy}

Despite comparable adequacy and complication rates, the EUS technique affords many advantages over percutaneous and transjugular approaches. While PCLB and TJLB have been associated with significant variation in sample yield, EUS strategies provide high-resolution imaging and access to sampling of both lobes of the liver, which may provide a more accurate representation of liver histology and reduced sampling error. ${ }^{10,20,44-46}$ Endosonography with Doppler capability allows for the avoidance of intrahepatic vessels and real-time imaging to navigate and avoid the bowel, gallbladder, and pleural space. ${ }^{12-14,44}$ Most importantly, EUSLB is able to achieve these results regardless of body habitus, whereas the percutaneous 


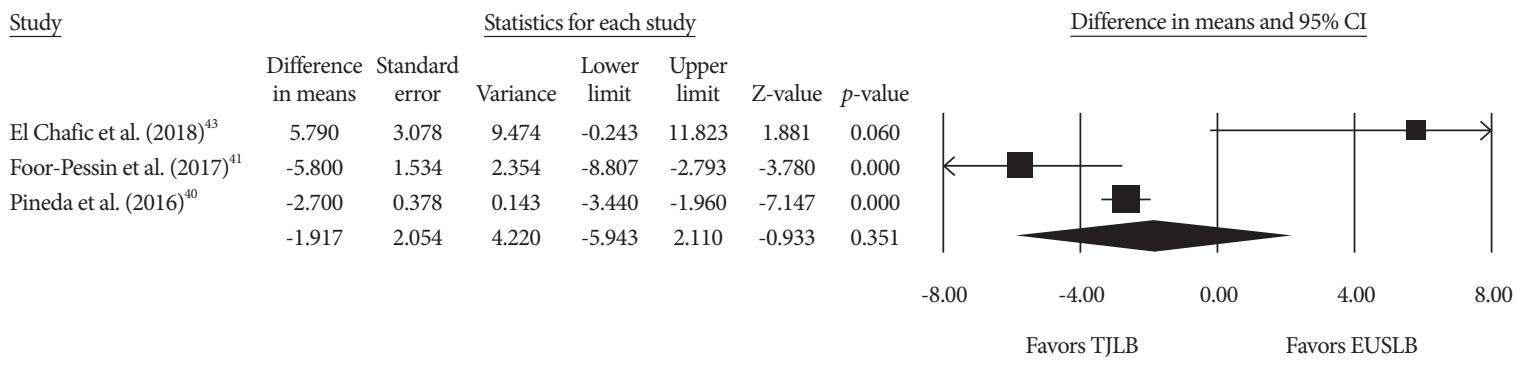

Complete portal triads: EUSLB vs. TJLB

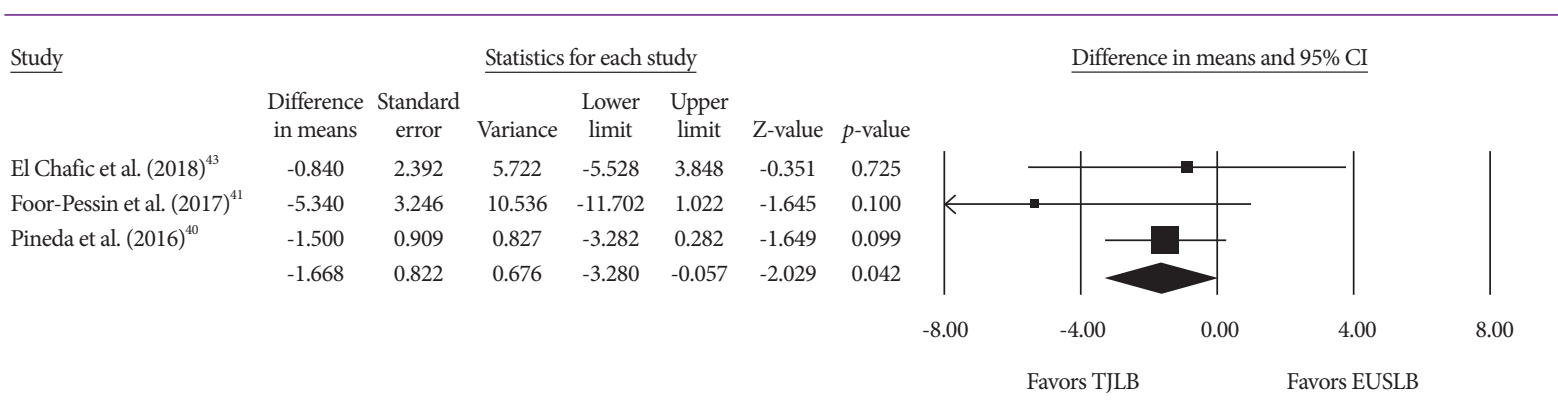

Total specimen length: EUSLB vs. TJLB

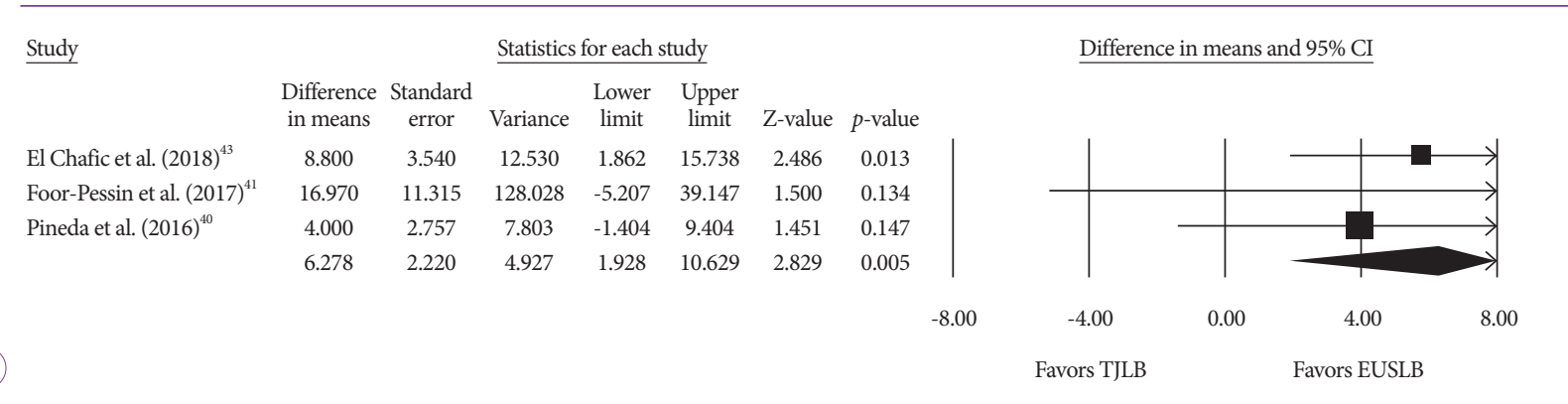

Meta analysis

Fig. 4. (A) Forest plot: comparison of length of longest piece for for endoscopic ultrasound-guided liver biopsy (EUSLB) versus transjugular liver biopsy (TJLB). (B) Forest plot: comparison of complete portal triads for EUSLB versus TJLB. (C) Forest plot: comparison of total specimen length for EUSLB versus TJLB. CI, confidence interval.

and, to a lesser extent, the transjugular routes, may be limited in obese patients. ${ }^{47}$ Furthermore, benefits of an EUS-guided approach include concomitant endoscopic esophageal variceal screening, gastric biopsy for Helicobacter pylori, and EUS imaging of the gallbladder and bile duct for evaluation of choledocholithiasis as a potential etiology of abnormal liver chemistries, in addition to the exclusion of ampullary and pancreas lesions. Despite these benefits, EUS may be limited by operator variability, requirement for deep sedation, procedure length, and associated monetary costs. ${ }^{20,40}$ However, the use of deep sedation may also beneficial, and may be appealing to the pa- tient, reducing any morbidity, pain, or anxiety associated with the procedure.

\section{Evolution of endoscopic ultrasound-guided liver biopsy technique}

The first published cases of EUSLB were described in 2007 using a novel Tru-Cut core biopsy needle (QuickCore; Cook Medical, Winston Salem, NC, USA). ${ }^{18,48}$ Since the introduction of the first EUSLB, many studies have been performed to determine the preferred needle. Schulman et al. sought to assess 6 needle types: $19 \mathrm{G}$ (SharkCore FNB [Medtronic], 
Expect FNA [Boston Scientific], and ProCore FNB [Echo Tip HD ProCore; Cook Medical]), 22 G (SharkCore FNB needle [Medtronic]), and percutaneous $18 \mathrm{G}$ (QuickCore [Cook Medical] and Coaxial Temno Needle [Care-Fusion, Waukegan, IL, USA]). ${ }^{17}$ This previous study found that the 19 G SharkCore FNB needle provided superior histologic yield compared to existing percutaneous needles. Therefore, we excluded a study that utilized the QuickCore and ProCore percutaneous needle from our comparative analysis based on the evidence of the reduced quality of these needles, current availability, and consideration of the needles utilized in routine clinical practice. $^{14}$

\section{Adequacy of biopsy specimen}

Currently, there are a variety of guidelines and recommendations that outline parameters for hepatic tissue adequacy regarding liver biopsy-each dependent upon the strategy or route of tissue acquisition. Cholongitas et al. previously performed a systematic review and meta-analysis of over 10,000 PCLBs and reported that a mean of 7.5 CPT and TSL of 17.7 $\mathrm{mm}$ were required for pathologic review. ${ }^{23}$ When obtained via the transjugular route, adequacy is defined as $6.5 \mathrm{CPT}$ and TSL of $12 \mathrm{~mm} .{ }^{10}$ Although an optimal definition of specimen adequacy for EUSLB has not yet been established, the American Association for the Study of Liver Diseases (AASLD) recommends $\geq 11 \mathrm{CPT}$ as the definition of adequacy, regardless of route of sampling. ${ }^{8}$ Perhaps more importantly, the AASLD guideline also utilizes TSL $>15 \mathrm{~mm}$ to define adequacy, albeit it considers that the ideal size is $30 \mathrm{~mm}{ }^{8,18}$ Although all routes of tissue sampling in our study achieved $\geq 11$ CPTs and TSL $>15 \mathrm{~mm}$, only EUSLB achieved the ideal TSL of $\geq 30 \mathrm{~mm}$.

\section{Limitations and strengths}

There are several limitations to this study. Although we assessed for heterogeneity as well as risk of bias, specific limitations include differences in patient population across multiple included modalities, as well as familiarity of the individual provider with the service (i.e., interventional radiology and gastroenterology) and with various techniques. Although each study evaluated all sampling techniques, there was significant heterogeneity among the included procedures (i.e., $I^{2}>50 \%$ ) and the variety of needle types utilized. Most importantly, the lack of a formal definition of EUSLB adequacy precludes consistency across centers and pathology providers. Similarly, while our cumulative analysis included EUS needle types no longer utilized in clinical practice, the use of a standard needle type is not verified across all institutions. Additionally, due to a paucity of data, the indication for liver biopsy could not be confirmed for individual cases, thereby limiting a subgroup analysis to evaluate for changes in efficacy or safety between different indications.

Furthermore, no randomized studies were included in this analysis and only two full-text manuscripts were included, thereby potentially increasing the selection bias. As most included studies were retrospective in design, there may have been a reason for EUSLB to be performed as opposed to other sampling routes-perhaps due to body habitus or other confounders that may artificially reduce or increase the efficacy or safety of the procedure. Publication bias was present in this meta-analysis; however, with the trim and fill method, the adequacy of EUSLB decreased from $93.51 \%$ to $81.94 \%$. Additionally, given the lack of current comparative data and expertise needed to perform EUSLB, the learning curve, or clinical acumen needed to perform an effective procedure, is unknownthereby potentially limiting the generalizability of our findings to centers with less familiarity or proven proficiency.

Despite these limitations, this study has several strengths. Most importantly, our meta-analysis using a random effects model to methodologically summarize all available data to evaluate feasibility, efficacy, and safety of EUSLB compared to PCLB and TJLB, with an emphasis on subgroup analysis analyzing needles only commonly used in current clinical practice. Although reporting bias of negative studies cannot be verified, small case series were eliminated to minimize inherent selection bias. Additionally, this systematic review and meta-analysis evaluated objective data, including biopsy adequacy, CPT, TSL, LLP, and adverse events for all included modalities. These findings, as highlighted in our systematic review and meta-analysis, are similar to previous cumulative systematic reviews and meta-analyses of EUSLB. Mohan et al. reported a histologic diagnosis rate of $93.9 \%$ and adverse event rate of $2.3 \%$ for EUSLB. ${ }^{20}$ These authors concluded that a $19 \mathrm{G}$ FNA needle provided optimal biopsy specimens. While recent published data by Diehl et al. suggests that heparin priming of EUS-FNA or FNB needles does not negatively affect cytologic or histologic interpretation, additional studies by similar authors have shown tissue adequacy to be higher for 19 G FNA versus $22 \mathrm{G}$ FNB needle among patients with chronic liver disease. ${ }^{49,50}$

To date, our study is the only meta-analysis to directly compare EUSLB, PCLB, and TJLB, including data from studies that evaluated all common approaches to obtain hepatic tissue. While TJLB provides an ability to simultaneously obtain portal pressure measures, EUS may be able to do the same in the near future. ${ }^{51,52}$ In fact, human studies have already been performed, and demonstrated a technical success rate of 100\% with no serious adverse events reported for EUS.$^{53,54}$ Moreover, this systematic review and meta-analysis specifically aimed to provide real-world data to simulate clinical practice through subgroup analysis that included only needle types that are cur- 
rently used in clinical practice. Despite the significant learning curve and inter-operator variability required to perform EUS, we are hopeful that the subgroup analysis will improve the generalizability of our results. While significant heterogeneity was noted in our meta-analysis and was not surprising given the cumulative nature of reporting results, the use of a prediction interval was employed to demonstrate the variability within real world clinical practice. ${ }^{28,55}$

In conclusions, EUSLB is a minimally invasive procedure that appears to be a safe and effective approach to obtain hepatic tissue. Although adequate needle size and tissue acquisition techniques for optimal EUS-FNA require further study, current data suggests that EUSLB is comparable to the alternative strategies of PCLB and TJLB. A cost-utility study to determine which modality is most cost-effective may be helpful to guide future decisions regarding diagnostic strategies. Future well-designed, prospective comparator studies are needed to determine which subset of patients may benefit most from EUSLB.

\section{Conflicts of Interest}

Marvin Ryou is a consultant for Medtronic/Covidien, Boston Scientific, Olympus, and Pentax. Harry R. Aslanian is a consultant for Boston Scientific, Olympus, Speaker for Cook, GI Supply. The other authors have no financial conflicts of interest.

\section{Author Contributions \\ Conceptualization: Thomas R. McCarty, Ahmad Najdat Bazarbashi, Mar- vin Ryou, Harry R. Aslanian, Thiruvengadam Muniraj \\ Formal analysis: TRM \\ Writing-original draft: TRM \\ Writing-review\&editing: ANB, MR, HRA, TM}

\section{ORCID}

Thomas R. McCarty: https://orcid.org/0000-0003-4517-5261

Ahmad Najdat Bazarbashi: https://orcid.org/0000-0002-5830-9938

Basile Njei: https://orcid.org/0000-0003-0714-4368

Marvin Ryou: https://orcid.org/0000-0001-8120-6497

Harry R. Aslanian: https://orcid.org/0000-0002-9126-0451

\section{REFERECES}

1. Bravo AA, Sheth SG, Chopra S. Liver biopsy. N Engl J Med 2001;344:495-500.

2. Tapper EB, Lok AS. Use of liver imaging and biopsy in clinical practice. N Engl J Med 2017;377:756-768.

3. Menghini G. One-second needle biopsy of the liver. Gastroenterology 1958;35:190-199.

4. van Leeuwen DJ, Wilson L, Crowe DR. Liver biopsy in the mid-1990s: questions and answers. Semin Liver Dis 1995;15:340-359.

5. Kirley K, Qato DM, Kornfield R, Stafford RS, Alexander GC. National trends in oral anticoagulant use in the United States, 2007 to 2011. Circ Cardiovasc Qual Outcomes 2012;5:615-621.

6. Grant A, Neuberger J. Guidelines on the use of liver biopsy in clinical practice. British Society of Gastroenterology. Gut 1999;45(Suppl 4):IV1-
IV11.

7. Dotter CT. Catheter biopsy. Experimental technic for transvenous liver biopsy. Radiology 1964;82:312-314.

8. Rockey DC, Caldwell SH, Goodman ZD, Nelson RC, Smith AD. Liver biopsy. Hepatology 2009;49:1017-1044.

9. Sheela H, Seela S, Caldwell C, Boyer JL, Jain D. Liver biopsy: evolving role in the new millennium. J Clin Gastroenterol 2005;39:603-610.

10. Kalambokis G, Manousou P, Vibhakorn S, et al. Transjugular liver biopsy--indications, adequacy, quality of specimens, and complications--a systematic review. J Hepatol 2007;47:284-294.

11. Schwarzer R, Ferlitsch A. Liver biopsy-transjugular. Clin Liver Dis (Hoboken) 2014;4:113-115.

12. Dewitt J, McGreevy K, Cummings O, et al. Initial experience with EUS-guided Tru-cut biopsy of benign liver disease. Gastrointest Endosc 2009;69(3 Pt 1):535-542.

13. Hollerbach S, Willert J, Topalidis T, Reiser M, Schmiegel W. Endoscopic ultrasound-guided fine-needle aspiration biopsy of liver lesions: histological and cytological assessment. Endoscopy 2003;35:743-749.

14. Nakanishi Y, Mneimneh WS, Sey M, Al-Haddad M, DeWitt JM, Saxena R. One hundred thirteen consecutive transgastric liver biopsies for hepatic parenchymal diseases: a single-institution study. Am J Surg Pathol 2015;39:968-976

15. Liberati A, Altman DG, Tetzlaff J, et al. The PRISMA statement for reporting systematic reviews and meta-analyses of studies that evaluate health care interventions: explanation and elaboration. Ann Intern Med 2009;151:W65-W94.

16. Stroup DF, Berlin JA, Morton SC, et al. Meta-analysis of observational studies in epidemiology: a proposal for reporting. Meta-analysis of observational studies in epidemiology (MOOSE) group. JAMA 2000;283:2008-2012.

17. Schulman AR, Thompson CC, Odze R, Chan WW, Ryou M. Optimizing EUS-guided liver biopsy sampling: comprehensive assessment of needle types and tissue acquisition techniques. Gastrointest Endosc 2017;85:419-426.

18. Mok SRS, Diehl DL. The role of EUS in liver biopsy. Curr Gastroenterol Rep 2019;21:6

19. Hirano T, Manabe T, Imanishi K, Ando K. Direct surface cooling of the exocrine pancreas in the rat. Br J Surg 1992;79:803-806.

20. Mohan BP, Shakhatreh M, Garg R, Ponnada S, Adler DG. Efficacy and safety of EUS-guided liver biopsy: a systematic review and meta-analysis. Gastrointest Endosc 2019;89:238-246.e3.

21. Standish RA, Cholongitas E, Dhillon A, Burroughs AK, Dhillon AP. An appraisal of the histopathological assessment of liver fibrosis. Gut 2006;55:569-578

22. Colloredo G, Guido M, Sonzogni A, Leandro G. Impact of liver biopsy size on histological evaluation of chronic viral hepatitis: the smaller the sample, the milder the disease. J Hepatol 2003;39:239-244.

23. Cholongitas E, Senzolo M, Standish R, et al. A systematic review of the quality of liver biopsy specimens. Am J Clin Pathol 2006;125:710-721.

24. DerSimonian R, Laird N. Meta-analysis in clinical trials. Control Clin Trials 1986;7:177-188.

25. Stuart A, Ord K. Kendall's advanced theory of statistics. 6th ed. London: Edward Arnold; 1994.

26. McCarty TR, Itidiare M, Njei B, Rustagi T. Efficacy of transoral incisionless fundoplication for refractory gastroesophageal reflux disease: a systematic review and meta-analysis. Endoscopy 2018;50:708-725.

27. McCarty TR, Njei B. Self-expanding metal stents for acute refractory esophageal variceal bleeding: a systematic review and meta-analysis. Dig Endosc 2016;28:539-547.

28. Riley RD, Higgins JP, Deeks JJ. Interpretation of random effects meta-analyses. BMJ 2011;342:d549.

29. Higgins JP, Thompson SG, Deeks JJ, Altman DG. Measuring inconsistency in meta-analyses. BMJ 2003;327:557-560.

30. Overton RC. A comparison of fixed-effects and mixed (random-effects) models for meta-analysis tests of moderator variable effects. Psychol 
Methods 1998;3:354-379.

31. Inoue T, Nishida T, Maekawa A, et al. Clinical features of post-polypectomy bleeding associated with heparin bridge therapy. Dig Endosc 2014;26:243-249.

32. Jadad AR, Moore RA, Carroll D, et al. Assessing the quality of reports of randomized clinical trials: is blinding necessary? Control Clin Trials 1996;17:1-12.

33. Gage BF, Waterman AD, Shannon W, Boechler M, Rich MW, Radford MJ. Validation of clinical classification schemes for predicting stroke: results from the national registry of atrial fibrillation. JAMA 2001;285:2864-2870.

34. Borenstein M, Higgins JP, Hedges LV, Rothstein HR. Basics of meta-analysis: $\mathrm{I}^{2}$ is not an absolute measure of heterogeneity. Res Synth Methods 2017;8:5-18.

35. Borenstein M, Hedges LV, Higgins JPT, Rothstein HR. Introduction to meta-analysis. Hoboken (NJ): Wiley; 2011.

36. Higgins JP, Thompson SG, Spiegelhalter DJ. A re-evaluation of random-effects meta-analysis. J R Stat Soc Ser A Stat Soc 2009;172:137-159.

37. Egger M, Davey Smith G, Schneider M, Minder C. Bias in meta-analysis detected by a simple, graphical test. BMJ 1997;315:629-634.

38. Easterbrook PJ, Berlin JA, Gopalan R, Matthews DR. Publication bias in clinical research. Lancet 1991;337:867-872.

39. Duval S, Tweedie R. Trim and fill: a simple funnel-plot-based method of testing and adjusting for publication bias in meta-analysis. Biometrics 2000;56:455-463.

40. Pineda JJ, Diehl DL, Miao CL, et al. EUS-guided liver biopsy provides diagnostic samples comparable with those via the percutaneous or transjugular route. Gastrointest Endosc 2016;83:360-365.

41. Foor-Pessin C, Bittner K, Kothari S, et al. Histologic yield of endoscopic ultrasound guided liver biopsy compared to percutaneous and transjugular approaches: a single-center retrospective review. Gastrointest Endosc 2017;85(5 Suppl):AB485-AB486.

42. Shahshahan M, Gertz H, Fakhreddine AY, et al. Endoscopic ultrasound-guided liver biopsy versus percutaneous and trans-jugular liver biopsy for evaluation of liver parenchyma. Gastrointest Endosc 2017;85(5 Suppl):AB490.

43. El Chafic AH, Mubarak MF, Shah J, et al. Endoscopic ultrasound-guided liver biopsy compared to percutaneous and transjugular liver biopsy: a tertiary center experience: category award (interventional endoscopy).
Am J Gastroenterol 2018;113:S421.

44. Diehl DL, Johal AS, Khara HS, et al. Endoscopic ultrasound-guided liver biopsy: a multicenter experience. Endosc Int Open 2015;3:E210-E215.

45. Maharaj B, Maharaj RJ, Leary WP, et al. Sampling variability and its influence on the diagnostic yield of percutaneous needle biopsy of the liver. Lancet 1986;1:523-525.

46. Gilmore IT, Burroughs A, Murray-Lyon IM, Williams R, Jenkins D, Hopkins A. Indications, methods, and outcomes of percutaneous liver biopsy in England and Wales: an audit by the British Society of Gastroenterology and the Royal College of Physicians of London. Gut 1995;36:437-441.

47. Singh P, Mukhopadhyay P, Bhatt B, et al. Endoscopic ultrasound versus CT scan for detection of the metastases to the liver: results of a prospective comparative study. J Clin Gastroenterol 2009;43:367-373.

48. Mathew A. EUS-guided routine liver biopsy in selected patients. Am J Gastroenterol 2007;102:2354-2355.

49. Diehl DL, Mok SRS, Khara HS, Johal AS, Kirchner HL, Lin F. Heparin priming of EUS-FNA needles does not adversely affect tissue cytology or immunohistochemical staining. Endosc Int Open 2018;6:E356-E362.

50. Mok SRS, Diehl DL, Johal AS, et al. Endoscopic ultrasound-guided biopsy in chronic liver disease: a randomized comparison of 19-G FNA and 22-G FNB needles. Endosc Int Open 2019;7:E62-E71.

51. Schulman AR, Thompson CC, Ryou M. EUS-guided portal pressure measurement using a digital pressure wire with real-time remote display: a novel, minimally invasive technique for direct measurement in an animal model. Gastrointest Endosc 2016;83:817-820.

52. Schulman AR, Thompson CC, Ryou M. Endoscopic ultrasound-guided direct portal pressure measurement using a digital pressure wire with real-time remote display: a survival study. J Laparoendosc Adv Surg Tech A 2017;27:1051-1054.

53. Huang JY, Samarasena JB, Tsujino T, et al. EUS-guided portal pressure gradient measurement with a simple novel device: a human pilot study. Gastrointest Endosc 2017;85:996-1001.

54. Samarasena JB, Huang JY, Tsujino T, et al. EUS-guided portal pressure gradient measurement with a simple novel device: a human pilot study. VideoGIE 2018;3:361-363.

55. Mohan BP, Adler DG. Heterogeneity in systematic review and meta-analysis: how to read between the numbers. Gastrointest Endosc 2019;89:902-903. 